\title{
Viscous Heating Effects on Heat Transfer Characteristics of an Explosive Fluid in a Converging Pipe
}

\author{
Sina Bagheri', Saeed Tavangar Roosta ${ }^{1 *}$, Amir Heidari $^{1}$ \\ ${ }^{1}$ Faculty of Chemistry and Chemical Engineering, Malek Ashtar University of Technology, Lavizan District, P. O. B. 15875-1774, \\ Tehran, Iran \\ * Corresponding author, e-mail: stavangar@mut.ac.ir
}

Received: 01 April 2020, Accepted: 18 May 2020, Published online: 11 June 2020

\begin{abstract}
Viscous dissipation is the production of heat due to the slip of fluid layers and can raise the temperature of the fluid that is affected by high shear stresses. This raise of temperature in fluids with explosive properties can cause the explosion during the processing. The present paper investigates the temperature distribution of an explosive fluid beside the wall of a converging tube. This study has been done by using the computational fluid dynamics and OpenFOAM software. The studied cases contain the fluid with two viscosities (50 and $500 \mathrm{~kg} / \mathrm{m} \times \mathrm{s}$ ) and two inlet conditions (constant and developed velocity profile). The results of this study show that at the end of a converging pipe, duo to the viscous dissipation effects, the temperature rise for high viscosity fluid is more intensive and this is a dangerous fact for high viscosity explosive fluids discharging. Also, it has been considered that the constant inlet velocity is safer in comparison with the developed profile, as the slope of temperature rise is less.
\end{abstract}

\section{Keywords}

viscous dissipation, computational fluid dynamics, explosive fluids, viscosity, convective heat transfer

\section{Introduction}

Over the past years, plenty of research has been done about the effects of the viscous dissipation phenomenon on the temperature distribution of fluids with high viscosity or fluids which pass the capillary and tapered tubes. The review of these researches revealed that the effects of viscous dissipation in laminar flow condition, have not been studied deeply yet. For fluids with high viscosity and low heat conduction, ignoring the viscous dissipation effects will make sensible errors in the calculations.

The work of Brinkman [1] appears to be the first theoretical work dealing with viscous dissipation. The temperature distribution in the entrance region of a circular pipe at the wall of which was maintained at either a constant temperature as that of the entering fluid or constant heat flux was examined. The highest temperatures were, not surprisingly, discovered to be localized in the wall region.

Tyagi [2] performed a wide study on the effect of viscous dissipation on the fully developed laminar forced convection in cylindrical tubes with an arbitrary cross-section and uniform wall temperature. Ou and Cheng [3] employed the separation of variables method to study the Graetz problem with finite viscous dissipation. They obtained the solution in the form of a series the eigenvalues and eigenfunctions of which satisfy the Sturm-Liouville system. The solution technique follows the same approach as that applicable to the classical Graetz problem and therefore suffers from the same weakness of poor convergence behavior near the entrance.

Lin et al. [4] showed that the effect of viscous dissipation was very relevant in the fully developed region if convective boundary conditions were considered. With these boundary conditions and if viscous dissipation was taken into account, the fully developed value of the Nusselt number was 48/5 for every value of the Biot number and of the other parameters. On the other hand, it is well known that, if a forced convection model with no viscous dissipation is employed, the fully developed value of the Nusselt number for convective boundary conditions depends on the value of the Biot number. Basu and Roy [5] analyzed the Graetz problem by taking account of viscous dissipation, but neglecting the effect of axial conduction. They showed that the effect of viscous dissipation could not be neglected when the wall temperature was uniform. For in-tube thermal conditions, Chaudhuri and Das [6] 
by using semi-analytical method, Teleszewski [7] for a tube with adiabatic wall and Newtonian fluid, Daghighi and Norouzi [8], and Dang [9] for a constant temperature wall condition, have been studied the effect of viscous dissipation in the thermal input region of a tube.

The effect of viscous dissipation in the thermal entrance region of slug flow forced convection in a circular duct was studied by Barletta and Zanchini [10]. The temperature field and the local Nusselt number were determined analytically for any prescribed axial distribution of wall heat flux, including uniform, linearly varying and exponentially varying heat fluxes.

Zanchini [11] analyzed the asymptotic behavior of laminar forced convection in a circular tube, for a Newtonian fluid at constant properties by taking into account the viscous dissipation effects. It was disclosed that particularly in the boundary conditions of uniform wall temperature and of heat transfer by convection to an external fluid yielded the same asymptotic behavior of the Nusselt number, namely $\mathrm{Nu}_{\infty}=48 / 5$. And therefore, he obviously stated that, for these boundary conditions, when the wall heat flux $q_{w}(x)$ tended to zero, i.e., the local Brinkman number $\operatorname{Br}(x)$ tended to infinity, it is completely wrong to neglect the effect of viscous dissipation on the asymptotic behavior of the forced convection problem.

Barletta [12] studied the effects of non-Newtonian Powerla fluid viscous dissipation on the asymptotic behavior of laminar and fully developed forced-flow temperature distributions within the cylindrical tube. In this study, the Nusselt number and asymptotic temperature distribution were calculated analytically for a constant wall temperature and for an external fluid displacement state. Daghighi and Norouzi [13] have investigated viscous dissipation effects in isothermal slits and tubes by using analytical solutions. Morini and Spiga [14] analytically determined the steady temperature distribution and the Nusselt numbers for a Newtonian incompressible fluid in a rectangular duct, in fully developed laminar flow with viscous dissipation, for any combination of heated and adiabatic sides of the duct.

Also the effects of viscous dissipation of forced laminar displacement for a Bingham fluid inside a cylindrical tube under different thermal boundary conditions have been investigated by Vradis et al. [15], Min et al. [16] and Khatyr et al. [17]. The temperature profile and Nusselt number are calculated in the developed temperature region for the axial distribution of the wall heat flux. Aydin [18, 19] investigated the temperature behavior of high viscosity Newtonian fluid inside the tube and with developed conditions at the inlet. In this study, two boundary conditions of constant wall temperature and constant heat flux at different Brinkman numbers on the development of fluid temperature inside the pipe are investigated.

The aim of the present work is to investigate the effect of viscous dissipation on a laminar convective explosive fluid flow in a converging pipe. The effects of different inlet and wall conditions on the temperature profile and the Nusselt number is obtained for the constant wall heat flux and the constant wall temperature thermal boundary conditions.

\section{Research methodology}

\subsection{Assumptions}

The numerical solution of the investigated problem is performed in 2D geometry and for the input fluid velocity, constant and developed profile have been considered. The simulations have been done with OpenFOAM ${ }^{1}$ software and the equations are in the steady-state form.

\subsection{Governing equations}

The three main equations of continuity, momentum and energy (Eqs. (1) to (3)) govern the fluid flow present in the problem [20]:

$$
\begin{aligned}
& \nabla \times(\rho \vec{U})=0 \\
& \nabla \times(\rho \vec{U} \vec{U})=\rho g-\nabla P+\nabla \times \tau_{i j}+S_{M} \\
& \rho(\nabla \times \vec{U})=\nabla \times(k \times \nabla T)+\varnothing .
\end{aligned}
$$

In Eqs. (1) to (3), density ( $\rho$ ), velocity vector ( $\vec{U})$, gravity acceleration $(g)$, pressure $(P)$, energy $(e)$, stress tension $\left(\tau_{i j}\right)$, heat transfer coefficient $(k)$ and $(\varnothing)$ is the viscous dissipation term. The above equations (Eqs. (1) to (3)) are the most general form of equations governing any type of fluid. It is also common for surface forces to be expressed as separate sentences in the equation of motion, to illustrate their role and to incorporate the effects of body forces into source expressions. But here, without taking into account the exact details of the body forces, the overall effect of these forces is only considered as a source term $\left(S_{M}\right)$ in the three dimensions of motion equations.

\subsection{Numerical simulation properties}

The geometry studied in this study is a circular converged channel in which the high viscosity explosive fluid begins to warm due to the shear stress on the channel wall. Fig. 1 presents the dimensions and boundary conditions of

1 The open field operation and manipulation 


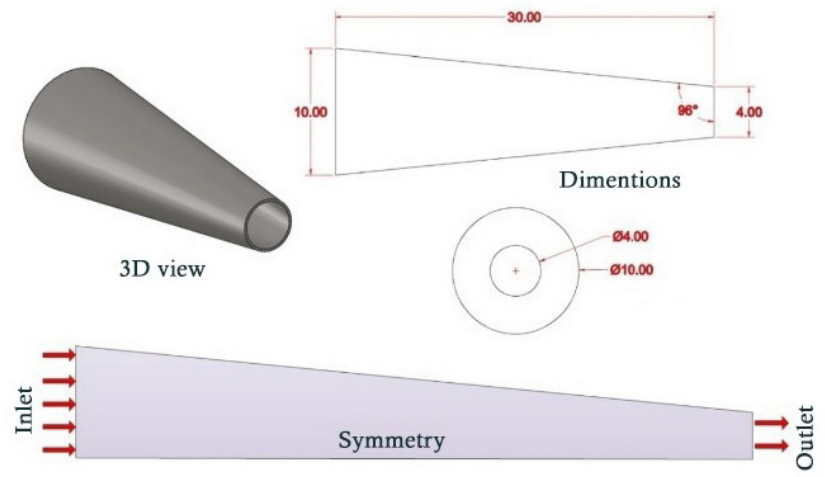

Fig. 1 Channel dimensions (cm) and boundary conditions

the investigated channel. Since the distribution of velocity and temperature in the studied channel is symmetric, it is possible to analyze the computational domain in two-dimensional terms by considering axial symmetry. In this case, due to reduced computational time and the possibility of increasing the number of grids, especially near the wall channel, the simulation results are very accurate. The number of meshes used here is 240,000 computational cells. The SIMPLE algorithm is chosen to solve the velocity-pressure equations and the QUICK method is used to discretize the governing equations. The studied cases typically employ convergence criteria of $10^{-5}$ to continuity and velocity residuals and $10^{-7}$ to energy residuals.

The desired fluid has a density of $1400 \mathrm{~kg} / \mathrm{m}^{3}$, $2000 \mathrm{~J} / \mathrm{kg} \times \mathrm{K}$ heat capacity and $0.27 \mathrm{~W} / \mathrm{m}^{2} \mathrm{~K}$ thermal conductivity and the inlet fluid temperature are set to $300 \mathrm{~K}$. Also, two different values (50 and $500 \mathrm{~kg} / \mathrm{m} \times \mathrm{s}$ ) for viscosity have been considered. The characteristics of the different cases that investigated in the present study are presented in Table 1.

\subsection{Numerical simulation results validation}

In order to validate the equations and models used in this simulation, the results presented by Aydin [18] have been used. In the work done by Aydin, the developed fluid flow enters a cylindrical tube. The effect of the Brinkman

Table 1 Characteristics of the different cases of the studied conditions

\begin{tabular}{lcc}
\hline Wall condition & Inlet Vel. & Case name \\
\hline Insulation & $1(\mathrm{~m} / \mathrm{s})$ & $\mathrm{L}-\mathrm{Vi}(500)-\mathrm{V}(1)$ \\
Insulation & $1(\mathrm{~m} / \mathrm{s})$ & $\mathrm{L}-\mathrm{Vi}(50)-\mathrm{V}(1)$ \\
Insulation & hagen_poiseuille & $\mathrm{L}-\mathrm{Vi}(500)-\mathrm{V}(\mathrm{P})$ \\
Insulation & hagen_poiseuille & $\mathrm{L}-\mathrm{Vi}(50)-\mathrm{V}(\mathrm{P})$ \\
$T=300 \mathrm{~K}$ & hagen_poiseuille & $\mathrm{L}-\mathrm{Vi}(500)-\mathrm{V}(\mathrm{P})-\mathrm{T}(300)$ \\
$T=250 \mathrm{~K}$ & hagen_poiseuille & $\mathrm{L}-\mathrm{Vi}(500)-\mathrm{V}(\mathrm{P})-\mathrm{T}(250)$ \\
\hline
\end{tabular}

number on the radial dimensionless temperature distribution in the developed thermal condition has been investigated by the analytical solution of the governing equations (Eqs. (1) to (3)).

The fluid flow studied by Aydin is thermal and velocity developed and it is assumed that the fluid properties (thermal conductivity, density and viscosity) are not temperature dependent. The fluid considered to be laminar and also the thermal conductivity along the axis has been ignored. The developed velocity profile of the tube in the developed state under the laminar flow condition known as the Hagen-Poiseuille equation (Eq. (4)):

$$
\frac{u}{u_{c}}=1-\left(\frac{r}{r_{0}}\right)^{2} \text {. }
$$

The energy equation (Eq. (5)) in the cylindrical coordinates for the tube is given by the viscous dissipation:

$u \frac{\partial T}{\partial Z}=\frac{v}{\operatorname{Pr}}\left[\frac{1}{r} \frac{\partial}{\partial r}\left(r \frac{\partial T}{\partial r}\right)+\frac{\partial^{2} T}{\partial X^{2}}\right]+\frac{v}{C_{p}}\left(\frac{\partial u}{\partial r}\right)^{2}$.

The second term in the right hand side is the viscous dissipation term. Given the axial symmetry conditions at the center of the tube, the thermal boundary condition at $r=0$ is written as follows:

$\frac{\partial T}{\partial r}=0$.

The boundary condition on the upper wall of the tube is defined as a constant amount of heat flux as follows:

$k \frac{\partial T}{\partial r}=q_{w}\left(r=r_{0}\right)$

The positive value of $\left(q_{w}\right)$ indicates wall heating and the negative value indicates the wall cooling. For the conditions of uniform heat flux in the wall, the first left-hand sentence of the energy equation can be defined as follows:

$\frac{\partial T}{\partial Z}=\frac{\partial T_{w}}{\partial Z}$

Also introducing the dimensionless longitudinal and temperature numbers as follows and applying them to the energy equation:

$$
\begin{aligned}
& R=\frac{r}{r_{0}}, \quad \theta=\frac{T_{w}-T}{T_{w}-T_{c}} \\
& \frac{1}{R} \frac{d}{d R}\left(R \frac{d \theta}{d R}\right)=a\left(1-R^{2}\right)-4 \mathrm{Br} R^{2},
\end{aligned}
$$


where $(a)$ and $(\mathrm{Br})$ are defined as follows:

$$
\begin{aligned}
& a=\frac{u_{c} r_{0}^{2}}{\alpha\left(T_{w}-T_{c}\right)} \frac{d T_{w}}{d z} \\
& \mathrm{Br}=\frac{\mu u_{c}^{2}}{k\left(T_{w}-T_{c}\right)} .
\end{aligned}
$$

To solve the dimensionless energy equation, Eq. (10), the dimensionless boundary conditions are defined as follows:

$\theta=1$ and $\frac{\partial \theta}{\partial R}=0$ at $R=0$

$\theta=0$ at $R=1$.

The solution of Eq. (10) based on the boundary conditions presented will be:

$\theta(R)=\left(1-\frac{4}{3} R^{2}+\frac{1}{3} R^{4}\right)+\frac{\mathrm{Br}}{3}\left(R^{2}-R^{4}\right)$.

The $(\mathrm{Br})$ value for the state where the boundary condition of the wall is defined by constant heat flux is given as follows:

$\mathrm{Br}_{q}=\frac{\mu u_{c}^{2}}{r_{0} q_{w}}$.

According to the above definition for the number (Br), the temperature distribution of the boundary condition of the wall with constant heat flux will be:

$$
\theta_{q}(R)=\left(\frac{3}{4}-R^{2}+\frac{R^{4}}{4}\right)+\mathrm{Br}_{q}\left(\frac{1}{2}-R^{2}+\frac{R^{4}}{2}\right) \text {. }
$$

Thus the analytical method used by Aydin was expressed. Therefore, Eq. (16) is used analytically to obtain the temperature distribution. On the other hand, to validate the numerical model used in the present study, with the results of this analytical solution presented by Aydin, the geometry and boundary conditions shown in Fig. 2 have been used.

The results of the dimensionless temperature distribution according to Eq. (16) with $\mathrm{Br}=2$ and $\mathrm{Br}=0.01$ values in the tube and wall boundary condition with constant heat flux are plotted in Fig. 3. In Fig. 3, the numerical

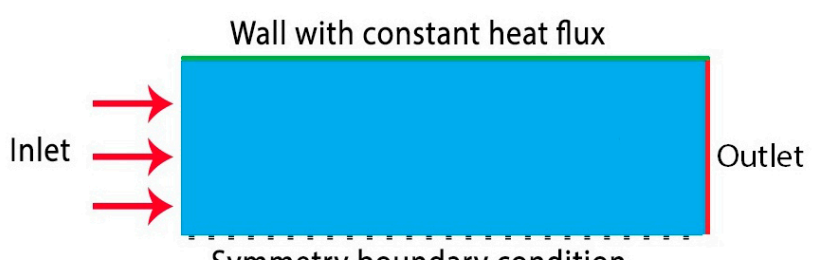

Symmetry boundary condition

Fig. 2 Geometry and boundary conditions simulation results along with the analytical solution are presented. Due to the proper matching of the numerical and analytical solution results, it can be concluded that the selected numerical method is suitable for solving the governing equations (Eqs. (1) to (3)) and can well predict the desired results regarding the distribution and increase of fluid temperature.

\section{Results}

\subsection{Investigation of the effect of inlet boundary conditions}

Fig. 4 shows the velocity distribution contour for an explosive fluid with high viscosity and constant inlet velocity. As can be seen in Fig. 4, as the flow approaches the outlet, the fluid velocity increases dramatically. Increasing the fluid velocity, increases the shear stress in the areas near the outlet and on the wall.

In Fig. 5, the temperature distribution of the explosive fluid passing through the channel is plotted. As shown in Fig. 5, due to the high tension between the fluid layers near the wall and the effect of viscosity on the energy equation (Eq. (3)), the wall temperature of the explosive fluid significantly increases to about 107 degrees.

In order to study the behavior of viscous dissipation and its effect on the explosive fluid temperature, shear stress and fluid temperature on the wall are plotted in Fig. 6. The results show that the shear stress has a significant

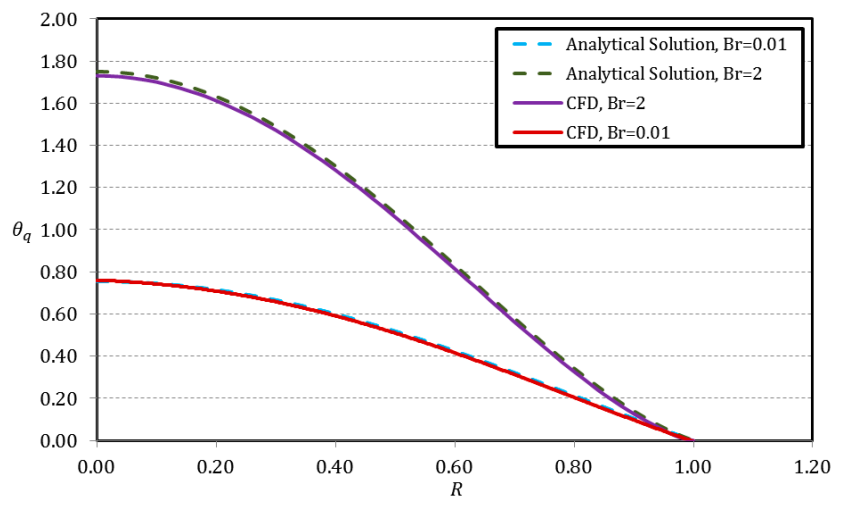

Fig. 3 Comparison of the results of analytical and numerical solution (CFD)

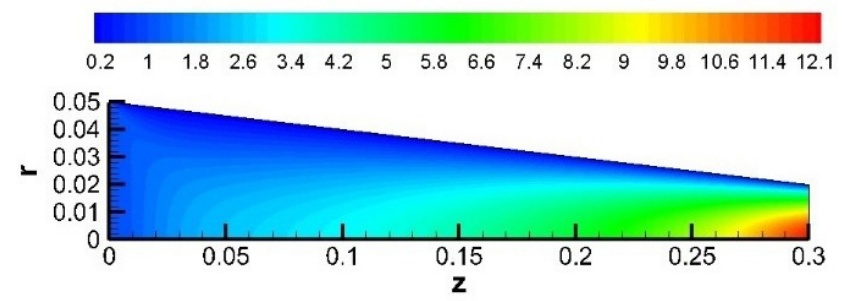

Fig. 4 Velocity distribution contour in case L-Vi (500) - V (1) $(\operatorname{Re}=0.028-1.1)$ 


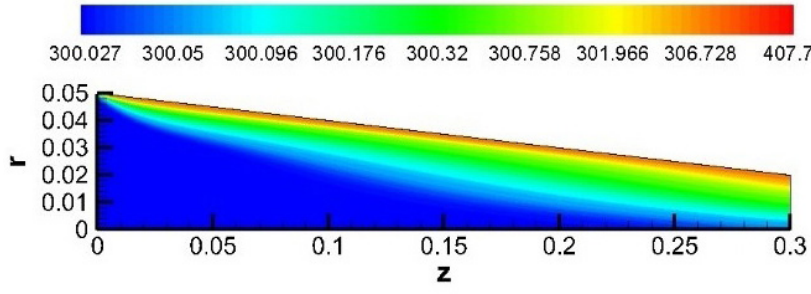

Fig. 5 Temperature distribution contour under the condition of case L-Vi (500) - V (1) $(\mathrm{Re}=0.028-1.1)$

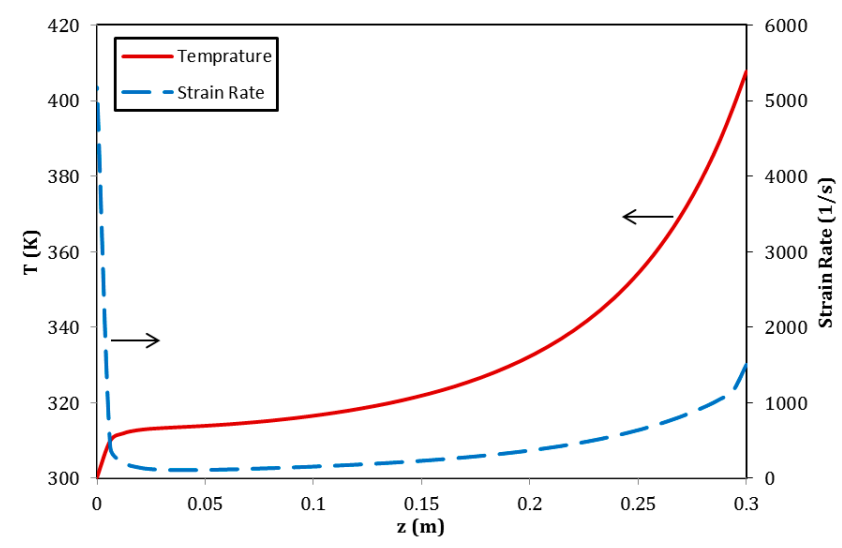

Fig. 6 Temperature and shear stress distribution on the wall in case L-Vi (500) - V (1) $(\mathrm{Re}=0.028-1.01)$

effect on the fluid temperature, with increasing shear stress near the outlet of the tube $(z>0.2 \mathrm{~m})$. It should also be noted that the highest rate of increase in temperature occurs at the tube entrance, due to the entry of an undeveloped linear and uniform flow into a converging tube, which causes very high shear stress at the inlet and on the wall.

In Fig. 7, the temperature distribution in the explosive fluid with low viscosity is plotted. Due to the high viscosity effect on the fluid temperature distribution, it is observed that in this case the maximum increase in explosive fluid temperature is $11 \mathrm{~K}$, which is about 96 degrees lower than the high viscosity fluid.

In Fig. 8, the comparison of temperature distribution for low and high viscosity (50 and $500 \mathrm{~kg} / \mathrm{m} \times \mathrm{s}$ ) and the laminar flow condition is plotted. This graph shows

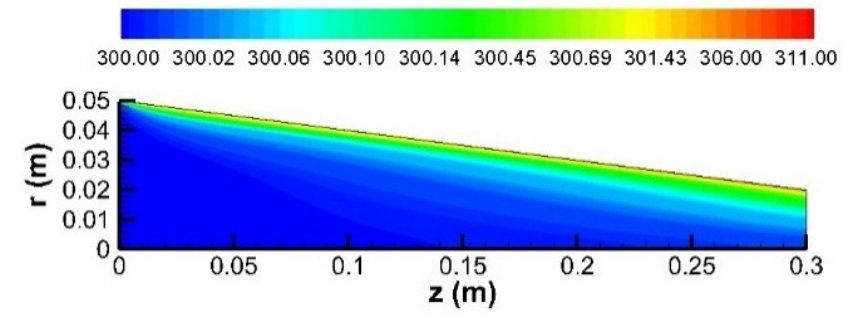

Fig. 7 Temperature distribution contour for the case L-Vi (50) - V (1) $(\operatorname{Re}=0.28-10.1)$

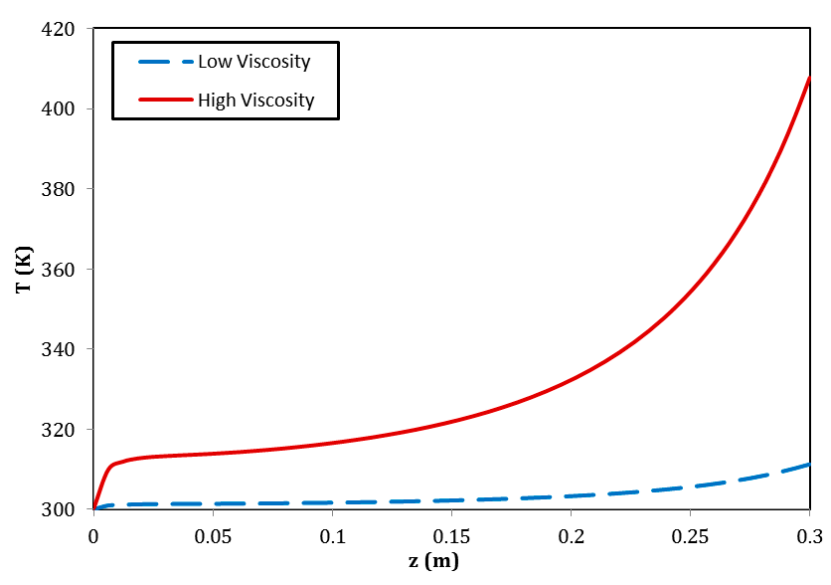

Fig. 8 Temperature distribution graph for low and high viscosity conditions for laminar flow model

some of the interesting points refers to the viscous dissipation effects. The viscous dissipation affects by viscosity and the second power of the local velocity gradient, simultaneously. At the positions near to the pipe inlet, the viscosity effects are prominent and the temperature raises steeper for the high viscosity case. The low viscosity case has a temperature growth with a gentle slope to near the positions of the pipe end. It seems that as the pipe is converging, duo to the increase in velocity, the second important factor (velocity gradient) plays the major role. In this district, the slope of two graphs increase, but the patterns are different and the temperature rise for the high viscosity case is more intensive. This is a dangerous fact for discharging the high viscosity explosive fluids and this effect which mainly relates to the viscous dissipation phenomena can lead to tragic accidents.

As shown in Table 1, in some numerical simulation cases, the flow velocity input is defined based on the Hagen-Poiseuille equation (Eq. (4)). Since the inlet velocity distribution in these cases is developed velocity distributions, we will investigate the developed conditions in the temperature distribution here. It is also worth noting that the velocity distribution in the developed condition is somehow assumed to equal its inlet mass flow rate with the inlet flow rate of the constant velocity condition. Consequently, the central velocity in the HagenPoiseuille equation (Eq. (4)) is equal to $u_{c}=2 \mathrm{~m} / \mathrm{s}$ by performing the calculations.

In Fig. 9, the temperature distribution contour and the wall temperature distribution diagram for the high viscosity fluid and the developed velocity condition is plotted. As can be seen, the maximum temperature in this state is $400.6{ }^{\circ} \mathrm{C}$ which is $7{ }^{\circ} \mathrm{C}$ lower than the high viscosity 


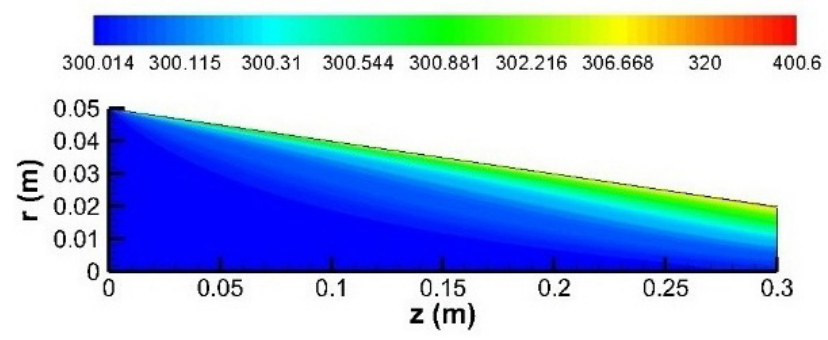

Fig. 9 Wall temperature contour for high viscosity fluid and developed velocity condition $(\mathrm{Re}=0.028-1.01)$

conditions at the constant input speed "L-Vi (500) - V (1)". Therefore, it can be concluded that the high viscosity fluid enters the channel under developed conditions, will significantly decrease the system temperature and also reduce the risk of fluid explosion.

Here in Fig. 10, the temperature distribution along the converging pipe for two different velocity inlet (constant and developed profile) is shown. It is interesting that after the constant velocity profile becomes kind of developed, its temperature grows less rapidly compared to the other case. It seems that the converging geometry is the reason for this phenomenon and maybe this is a good result for explosive fluid. Especially by attending the fact that in practical application, it is rare that the shape of velocity profile can be controlled. So it can be concluded that for explosive fluid applications, the constant velocity inlet is a safer and more available choice.

\section{2 Investigation of the effect of wall temperature on the temperature distribution of explosive fluid}

Since the passage of explosive fluid through the pipe increases its temperature, especially at the end of it, in this section we investigate the effect of pipe wall temperature

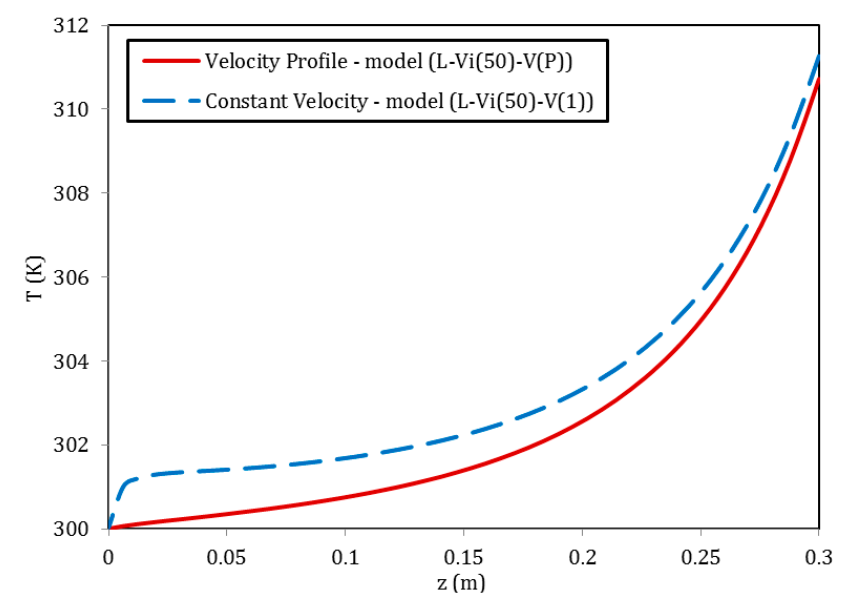

Fig. 10 Comparison diagram of wall temperature for two steady-state and low-viscosity cases $(\mathrm{Re}=0.28-10.1)$ on the fluid temperature distribution based on the developed velocity input, so "L-Vi (500) - V (P) - T (300)" and "L-Vi (500) - V (P) - T (300)" cases are discussed here.

In Fig. 11, the distribution of the pipe wall temperature in three different wall conditions, including the insulation condition, namely the "L-Vi (500) - V (P)" case and the $300 \mathrm{~K}$ wall temperature, namely the "L-Vi (500) - V (P) - T (300)" case is plotted. As can be seen in Fig. 11, a model in which the wall temperature is set at $300{ }^{\circ} \mathrm{C}$, in the studied sections ( $z=0.15$ and $z=0.30 \mathrm{~m}$ respectively) predicts lower temperatures compared to insulation wall conditions.

In Fig. 12, the wall temperature is kept constant at $250 \mathrm{~K}$ and the temperature distribution in two radial sections $(z=0.15$ and $z=0.30 \mathrm{~m}$ ) has been compared to the tube with constant wall temperature of $300 \mathrm{~K}$. As can be seen in Fig. 12, at the end of the tube, there is a maximum of 4 degrees difference between operating conditions. Therefore, it seems that 50 degree decrease

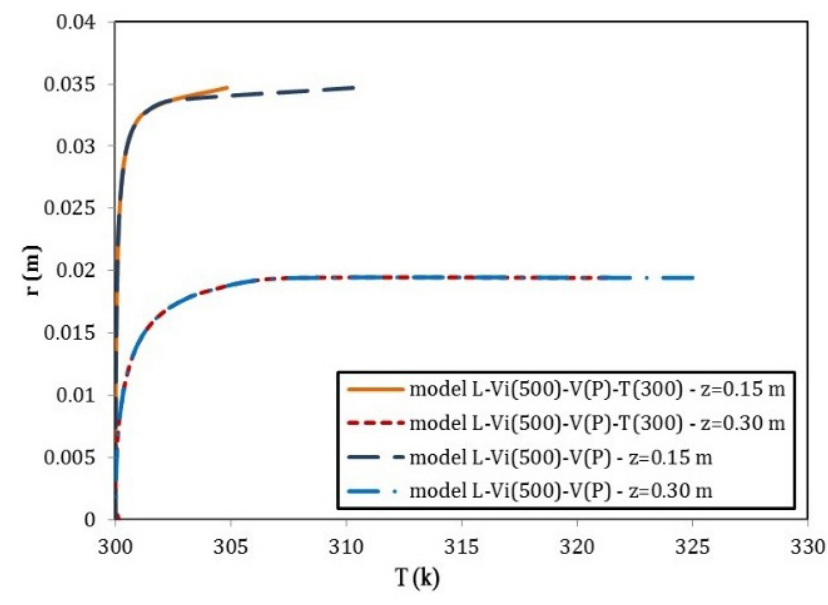

Fig. 11 Radial temperature distribution in a laminar flow, insulation wall and wall at $300 \mathrm{~K}$

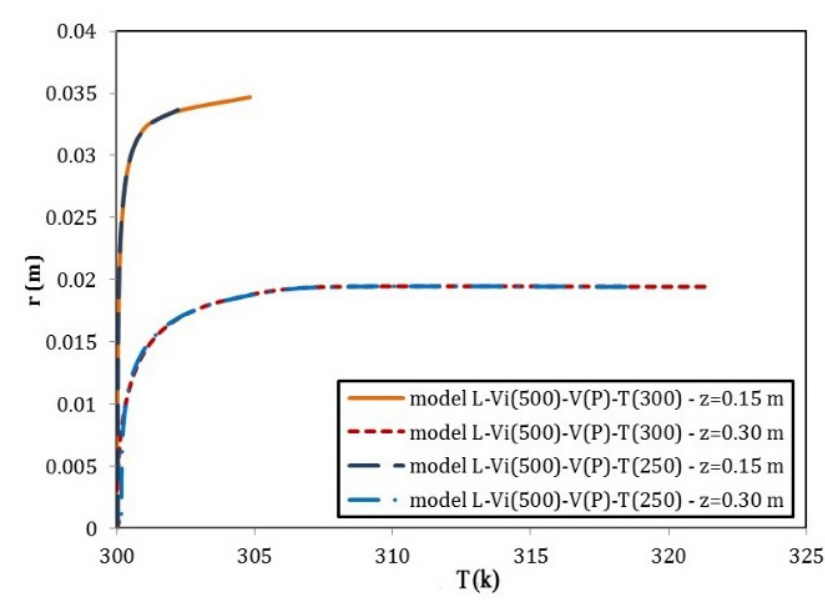

Fig. 12 Radial temperature distribution in a laminar flow, wall at 300 and $250 \mathrm{~K}(\operatorname{Re}=0.028-1.01)$ 
in wall temperature in the "L-Vi (500) - V (P) - T (250)" case compared to the "L-Vi (500) - V (P) - T (300)" has not been able to significantly reduce the temperature of the explosive fluid. However, it should be noted that in the case of "L-Vi (500) - V (P) - T (250)" relative to the insulation wall case "L-Vi (500) - V (P)", the fluid temperature indicates 8 degrees difference that can have a significant impact on process safety.

\section{Conclusion}

In this study, first, a review of researches on high viscosity fluid modeling has presented. Then the numerical model used in the present simulation with its equations (Eqs. (1) to (3)) has fully studied. The investigated equations are continuity, momentum and energy equations (together with the viscous dissipation effects). The boundary conditions used in numerical simulation have also presented.

In order to validate the numerical simulation results, the high viscosity fluid temperature distribution was compared with the analytical solution of the flow inside a pipe. The results of this section showed very good agreement between the numerical simulation results and the results of the analytical solution. After validation of the numerical simulation results, the explosive fluid flow within the converged tube was investigated by numerical validation method. Studies have been carried out to investigate

\section{References}

[1] Brinkman, H. C. "Heat effects in capillary flow I", Applied Scientific Research, 2(1), Article Number: 120, 1951. https://doi.org/10.1007/BF00411976

[2] Tyagi, V. P. "Laminar Forced Convection of a Dissipative Fluid in a Channel", Journal of Heat Transfer, 88(2), pp. 161-167, 1966. https://doi.org/10.1115/1.3691501

[3] Ou, J. W., Cheng, K. C. "Viscous dissipation effects on thermal entrance region heat transfer in pipes with uniform wall heat flux", Applied Scientific Research, 28(1), pp. 289-301, 1973. https://doi.org/10.1007/BF00413074

[4] Lin, T. F., Hawks, K. H., Leidenfrost, W. "Analysis of viscous dissipation effect on thermal entrance heat transfer in laminar pipe flows with convective boundary conditions", Wärme - und Stoffübertragung, 17(2), pp. 97-105, 1983. https://doi.org/10.1007/BF01007225

[5] Basu, T., Roy, D. N. "Laminar heat transfer in a tube with viscous dissipation", International Journal of Heat and Mass Transfer, 28(3), pp. 699-701, 1985.

https://doi.org/10.1016/0017-9310(85)90191-7 different operating plans to find fluid temperature distribution under the low and high viscosity conditions, developed or constant inlet velocity based on laminar flow model. Investigation of the results of high viscosity shows that as the pipe is converging, duo to the increase in velocity, the viscous dissipation effects become more effective. In this district, the temperature rise for the high viscosity case is more intensive. This is a dangerous fact for discharging the high viscosity explosive fluids and this phenomena can lead to tragic accidents.

Also study the shape of the inlet velocity profile shows that after the constant velocity profile becomes kind of developed, its temperature grows less rapidly compared to the other case. It can be concluded that for explosive fluid applications, the constant velocity inlet is a safer and more available choice.

In order to investigate the effect of wall cooling on the in-tube fluid temperature distribution, the tube wall was studied at 300 and $250 \mathrm{~K}$ under laminar flow conditions. The results showed that the wall temperature of the fluid decreased to a maximum of 8 degrees. However, the temperature difference of explosive fluid in the conditions of 300 and $250 \mathrm{~K}$ wall temperature is maximum 4 degrees, which can be determined more precisely by the operating costs and fluid conditions for the wall boundary conditions.

[6] Chaudhuri, S., Das, P. K. "Semi-Analytical Solution of the Heat Transfer Including Viscous Dissipation in the Steady Flow of a Sisko Fluid in Cylindrical Tubes", Journal of Heat Transfer, 140(7), Article Number: 071701, 2018.

https://doi.org/10.1115/1.4039352

[7] Teleszewski, T. J. "Viscous dissipation effect on laminar forced convection in a circular duct with adiabatic walls - preliminary numerical investigation", In: XI International Conference on Computational Heat, Mass and Momentum Transfer (ICCHMT 2018), Cracow, Poland, 2018, Article Number: 03016.

https://doi.org/10.1051/matecconf/201824003016

[8] Daghighi, S. Z., Norouzi, M. "Analysis of forced convection of Phan-Thien-Tanner fluid in slits and tubes of constant wall temperature with viscous dissipation", Journal of the Brazilian Society of Mechanical Sciences and Engineering, 41(11), Article Number: 480, 2019. https://doi.org/10.1007/s40430-019-1992-4

[9] Dang, V. D. "Heat Transfer of Power Law Fluid at Low Peclet Number Flow", Journal of Heat Transfer, 105(3), pp. 542-549, 1983. https://doi.org/10.1115/1.3245619 
[10] Barletta, A., Zanchini, E. "Forced convection in the thermal entrance region of a circular duct with slug flow and viscous dissipation", International Journal of Heat and Mass Transfer, 40(5), pp. 1181-1190, 1997.

https://doi.org/10.1016/0017-9310(96)00212-8

[11] Zanchini, E. "Effect of viscous dissipation on the asymptotic behaviour of laminar forced convection in circular tubes", International Journal of Heat and Mass Transfer, 40(1), pp. 169-178, 1996.

https://doi.org/10.1016/S0017-9310(96)00076-2

[12] Barletta, A. "Fully developed laminar forced convection in circular ducts for power-law fluids with viscous dissipation", International Journal of Heat and Mass Transfer, 40(1), pp. 15-26, 1996. https://doi.org/10.1016/S0017-9310(96)00094-4

[13] Daghighi, S. Z., Norouzi, M. "Effects of viscous dissipation on heat convection of viscoelastic flow inside isothermal channels and tubes", Korea-Australia Rheology Journal, 30(4), pp. 273-292, 2018. https://doi.org/10.1007/s13367-018-0026-6

[14] Morini, G. L., Spiga, M. "Nusselt Numbers in Rectangular Ducts with Laminar Viscous Dissipation", Journal of Heat Transfer, 121(4), pp. 1083-1087, 1999. https://doi.org/10.1115/1.2826061

[15] Vradis, G. C., Dougher, J., Kumar, S. "Entrance pipe flow and heat transfer for a Bingham plastic", International Journal of Heat and Mass Transfer, 36(3), pp. 543-552, 1993. https://doi.org/10.1016/0017-9310(93)80030-X
[16] Min, T., Yoo, J. Y., Choi, H. "Laminar convective heat transfers of a Bingham plastic in a circular pipe - I. Analytical approach thermally fully developed flow and thermally developing flow (the Graetz problem extended)", International Journal of Heat and Mass Transfer, 40(13), pp. 3025-3037, 1997. https://doi.org/10.1016/S0017-9310(96)00362-6

[17] Khatyr, R., Ouldhadda, D., Il Idrissi, A. "Viscous dissipation effects on the asymptotic behaviour of laminar forced convection for Bingham plastics in circular ducts", International Journal of Heat and Mass Transfer, 46(4), pp. 589-598, 2003. https://doi.org/10.1016/S0017-9310(02)00331-9

[18] Aydin, O. "Effects of viscous dissipation on the heat transfer in forced pipe flow. Part 1: both hydrodynamically and thermally fully developed flow", Energy Conversion and Management, 46(5), pp. 757-769, 2005.

https://doi.org/10.1016/j.enconman.2004.05.004

[19] Aydin, O. "Effects of viscous dissipation on the heat transfer in a forced pipe flow. Part 2: Thermally developing flow", Energy Conversion and Management, 46(18-19), pp. 3091-3102, 2005. https://doi.org/10.1016/j.enconman.2005.03.011

[20] Cavazzuti, M. "Viscous heating effects on heat transfer characteristics of laminar compressible channel flow", International Journal of Heat and Mass Transfer, 153, Article Number: 119608, 2020. https://doi.org/10.1016/j.ijheatmasstransfer.2020.119608 